\title{
FACTORES DE RIESGO ASOCIADOS A PIE DIABÉTICO EN EL HOSPITAL PNP LUIS N. SAENZ, AÑO 2017
}

\author{
RISK FACTORS ASSOCIATED TO DIABETIC FOOT AT LUIS N. SAENZ PNP HOSPITAL, YEAR 2017
}

Yajaira Arribasplata-Espinoza ${ }^{1, a}$, Consuelo Luna-Muñoz $2, b$

\begin{abstract}
RESUMEN
Introducción: El pie diabético es una de las complicaciones más graves y frecuentes de la diabetes mellitus, por tal motivo es importante conocer los factores de riesgo que lo desencadenan. Objetivo: Determinar los factores de riesgo asociados a pie diabético en el hospital PNP Luis N. Sáenz, año 2017. Métodos: Estudio observacional, cuantitativo, retrospectivo, analítico, de casos y controles. La muestra la conformó 165 pacientes (55 casos y 110 controles) atendidos en el servicio de endocrinología en el año 2017. Se estudiaron factores de riesgo, modificables y no modificables, asociados al desarrollo de pie diabético; se recogió la información de las historias clínicas mediante una ficha de recolección de datos, la cual fue analizada mediante el programa SPSS versión 25.0 utilizando los modelos estadísticos de Chi cuadrado y el odds ratios con un nivel de significancia menor a $0.05 \%$ y un intervalo de confianza de 95\%. Resultados: Los factores de riesgo asociados a pie diabético son tiempo de enfermedad ( $\mathrm{p}: 0,000$; OR: 12.77; IC: 4.12 - 39.60), hemoglobina glicosilada mal controlada (p: 0.029; OR: 4.55; IC: 1.17 - 17.68), onicomicosis (p: 0.014; OR: 7.10; IC: 1.48 - 34.07); antecedente de tabaquismo (p: 0.031; OR: 2.66; IC: 1.09 - 6.34) y enfermedad renal crónica (p: 0.047; OR: 3.023; IC: 1.01 - 9.0). Conclusión: Se concluye que hay asociación estadística significativa entre tiempo de enfermedad, hemoglobina glicosilada mal controlada, onicomicosis, enfermedad renal crónica y antecedente de tabaquismo, con desarrollar pie diabético.
\end{abstract}

Palabras clave: Pie diabético; Factores de riesgo no modificables; Factores de riesgo modificables. (fuente: DeCS BIREME)

\begin{abstract}
Introduction: Diabetic foot is one of the most serious and frequent complications of diabetes mellitus, for this reason it is important to know the risk factors that trigger it. Objective: To determine the risk factors associated with diabetic foot in the PNP Luis N. Sáenz Hospital, 2017. Methods: Observational, retrospective, analytical, case and control study of 165 patients ( 55 cases and 110 controls) treated in the endocrinology service in 2017. Modifiable and non-modifiable risk factors associated with the development of diabetic foot were studied; the information of the clinical histories was collected through a data collection form, which was analyzed by means of the SPSS program version 25.0 using the statistical models of Chi square and the odds ratios with a level of significance less than $0.05 \%$ and a range of $95 \%$ confidence. Results: The risk factors associated with diabetic foot are disease time (p: 0.000, OR: 12.77, Cl: 4.12 - 39.60); poorly controlled glycosylated hemoglobin (p: 0.029, OR: 4.55, Cl: 1.17 - 17.68); Onychomycosis (p: 0.014, OR: 7.10, $\mathrm{Cl}: 1.48$ - 34.07); antecedent of smoking (p: 0.031 , OR: $2.66, \mathrm{Cl}: 1.09-6.34$ ) and chronic kidney disease (p: 0.047 , OR: $3.023, \mathrm{Cl}: 1.01-9.0$ ). Conclusion: We conclude that there is a statistically significant association between time of illness, poorly controlled glycosylated hemoglobin, onychomycosis, chronic kidney disease, history of smoking and developing diabetic foot.
\end{abstract}

Key words: Diabetic foot; Non-modifiable risk factors; Modifiable risk factors. (source: MeSH NLM) 


\section{INTRODUCCIÓN}

La Organización Mundial de la Salud (OMS) en el año 2016 reportó que, en el mundo la población de adultos con diabetes mellitus en el año 2014 ascendía a 422 millones. Según encuesta Demográfica y de Salud Familiar 2016 (ENDES), la diabetes mellitus tipo 2 a nivel nacional es un problema de salud pública, debido a su prevalencia (3.2\%) y al gasto que genera el tratamiento y manejo de las complicaciones ${ }^{1,2}$. El pie diabético suma largas permanencias hospitalarias en contraste con cualquier otra complicación de la diabetes mellitus tipo 2 , con morbilidad y mortalidad significativa ${ }^{3}$.

Los factores asociados a pie diabético se dividen en dos grandes grupos, en no modificables y modificables. En este estudio se analizaron los factores de riesgo no modificables: sexo, edad y tiempo de evolución de la enfermedad mayor a diez años y los factores modificables como hemoglobina glicosilada mayor a $7.5 \%$, antecedente de tabaquismo, onicomicosis, obesidad, sobrepeso, enfermedad vascular periférica, neuropatía periférica, presencia de hipertensión arterial, enfermedad renal crónica y dislipidemia.

El pie diabético, sin duda el día de hoy, representa una de las complicaciones más graves y frecuentes de la diabetes mellitus 2, por tal motivo es importante determinar los factores de riesgo asociados a pie diabético en el hospital PNP Luis N. Sáenz durante el año 2017.

\section{MÉTODOS}

\section{Tipo y diseño de investigación}

El diseño de investigación del presente estudio es tipo observacional, cuantitativo, retrospectivo, analítico, de casos y controles, se desarrolló en el contexto del
IV CURSO - TALLER DE TITULACIÓN POR TESIS según enfoque y metodología publicada4.

\section{Población y muestra}

El estudio incluyó a los pacientes con diagnóstico de diabetes mellitus tipo 2 atendidos en el servicio de endocrinología del hospital PNP Luis N. Sáenz durante los meses de enero a diciembre del 2017. La muestra estuvo conformada por 165 pacientes, 55 casos con diagnóstico de diabetes mellitus tipo 2 y pie diabético y 110 controles con diagnóstico de diabetes mellitus tipo 2 sin pie diabético controles, con una relación de 1: 2 .

\section{Técnica de procesamiento y análisis de datos}

Se empleó la calculadora "IMIN" (IV Curso - Taller de Titulación por tesis) y en base a la bibliografía revisada se consideró una incidencia de $9.8 \%$, y odds ratio de 3.8, nivel de significancia estadística menor a $0.05 \%$ y un intervalo de confianza de $95 \%$.

La información se obtuvo de las historias clínicas de los pacientes y se registró en una hoja de recolección de datos, se empleó el programa IBM SPSS Statistics 25.0 para el procesamiento de los mismos. Se realizó estadística descriptiva, análisis bivariado (Chi cuadrado y Odds ratio) y regresión logística de las variables de interés.

\section{RESULTADOS}

Se evaluaron las siguientes variables: sexo, edad avanzada (mayor de 60 años), índice de masa corporal (sobrepeso, obesidad), tiempo de enfermedad mayor de diez años, mal control (hemoglobina glicosilada $>7.5 \%)$, enfermedad vascular perifèrica, neuropatía periférica, antecedemte de tabaquismo, onicomicosis, comorbilidades (hipertensión arterial, enfermedad renal crónica, dislipidemia).

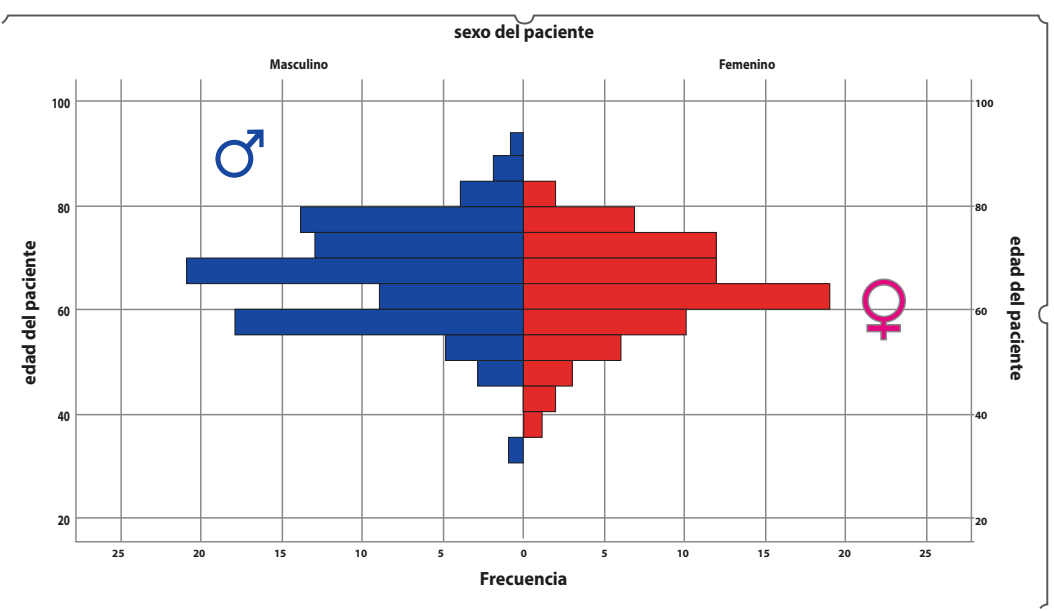

Fuente: Historias clínicas Hospital de Policía Luis N. Sáenz.

Gráfico 1. Pirámide edad / sexo de pacientes atendidos en el servicio de endocrinología del Hospital de Policía, enero- diciembre de 2017. 
Con respecto a la edad de los pacientes según grupos etarios, el grupo que predominó es el adulto mayor (>=60 años) con 116 pacientes (70.3\%), el grupo etario $<60$ años estuvo conformado por 49 pacientes (29.7\%).

En ambos sexos las edades fluctuaron entre los 60 y 80 años y fueron los pacientes de sexo masculino los que tuvieron edades extremas. La edad mínima fue de 32 años y la edad máxima de 93 años, se obtuvo una media de 65.02 años, una moda de 69 años y una mediana de 65 años. En lo referente a la variable sexo, se encontró predominancia del sexo masculino 91 (55.2\%), en comparación al femenino 74 (44.8\%). Gráfico 1.

Tabla 1. Distribución del índice de masa corporal.

FRECUENCIA

3

26

92

39

4

1

\section{PORCENTAJE}

$1.8 \%$

$15.8 \%$

$55.8 \%$

$23.6 \%$

$2.4 \%$

$0.6 \%$

Obesidad tipo III

$>40$

entre $25-29.9$ y 39 pacientes con IMC entre 30-34.9,

En la categoría de índice de masa corporal encontramos que, del total de pacientes, 131 (79.4\%) presentaron respectivamente. (Tabla 1). sobrepeso y obesidad tipo I, 92 pacientes con IMC

Tabla 2. Asociación entre factores de riesgo no modificables y pie diabético.

\begin{tabular}{|c|c|c|c|c|c|c|}
\hline \multicolumn{2}{|c|}{ VARIABLE } & \multirow{2}{*}{$\begin{array}{l}\text { PIE DIABÉTICO } \\
29(31.9 \%)\end{array}$} & \multirow{2}{*}{$\begin{array}{c}\text { NO PIE } \\
\text { DIABĖTICO } \\
62(68.1 \%)\end{array}$} & \multirow{2}{*}{$\begin{array}{c}\text { P VALOR } \\
\\
0.65\end{array}$} & \multirow{2}{*}{$\begin{array}{c}\text { IC } 95 \% \\
0.45-1.65\end{array}$} & \multirow{2}{*}{$\begin{array}{l}\text { OR } \\
0.86\end{array}$} \\
\hline Sexo & Masculino & & & & & \\
\hline & Femenino & $26(35.1 \%)$ & $48(64.9 \%)$ & & & \\
\hline \multirow{3}{*}{ Edad } & $>60$ años & 42 (76.4\%) & 74 (67.3\%) & 0.22 & $0.30-1.33$ & 0.63 \\
\hline & $<60$ años & $13(23.6 \%)$ & $36(32.7 \%)$ & & & \\
\hline & Total & $55(100 \%)$ & $110(100 \%)$ & & & \\
\hline Tiempo de enfermedad & > 10 años & 39 (70.9\%) & $57(51.8 \%)$ & 0.019 & $1.13-4.52$ & 2.26 \\
\hline
\end{tabular}

De los factores de riesgo no modificables, el tiempo de enfermedad mayor a 10 años tiene 2,26 veces más riesgo de desarrollar pie diabético, la asociación es estadísticamente significativa (p: 0.019, OR: 2.26, IC: 1.13-4.52). 
Tabla 3. Asociación entre factores de riesgo modificables y pie diabético.

\begin{tabular}{|c|c|c|c|c|c|c|}
\hline VARIABLE & & $\begin{array}{c}\text { PIE } \\
\text { DIABĖTICO }\end{array}$ & $\begin{array}{c}\text { NO PIE } \\
\text { DIABĖTICO }\end{array}$ & $\begin{array}{c}\text { P } \\
\text { VALOR }\end{array}$ & IC 95\% & OR \\
\hline \multirow[t]{3}{*}{ Hemoglobina glicosilada } & Mal control & $52(94.5 \%)$ & $81(73.6 \%)$ & 0.001 & $1.79-21.41$ & 6.2 \\
\hline & Buen control & $3(5.5 \%)$ & $29(26.4 \%)$ & & & \\
\hline & Total & $55(100 \%)$ & $110(100 \%)$ & & & \\
\hline \multirow[t]{3}{*}{ Onicomicosis } & Presente & $52(94.5 \%)$ & $86(78.2 \%)$ & 0.007 & $1.38-16.86$ & 4.83 \\
\hline & Ausente & $3(5.5 \%)$ & $24(21.8 \%)$ & & & \\
\hline & Total & $55(100 \%)$ & $110(100 \%)$ & & & \\
\hline \multirow{3}{*}{$\begin{array}{l}\text { Enfermedad vascular } \\
\text { periférica }\end{array}$} & Presente & 31 (56.4\%) & 37 (33.65) & 0.005 & $1.31-4.94$ & 2.54 \\
\hline & Ausente & $24(43.65)$ & $73(66.4 \%)$ & & & \\
\hline & Total & $55(100 \%)$ & $110(100 \%)$ & & & \\
\hline \multirow[t]{3}{*}{ Neuropatía periférica } & Presente & $38(69.15)$ & $48(43.6 \%)$ & 0.002 & $1.45-5.72$ & 2.88 \\
\hline & Ausente & 17 (30.9\%) & $62(56.4 \%)$ & & & \\
\hline & Total & 55 (100\%) & 110 (100\%) & & & \\
\hline \multirow[t]{3}{*}{$\begin{array}{l}\text { Antecedente de } \\
\text { tabaquismo }\end{array}$} & Presente & $43(78.2 \%)$ & $59(53.6 \%)$ & 0.002 & $1.47-6.50$ & 3.09 \\
\hline & Ausente & $12(21.8 \%)$ & $51(46.4 \%)$ & & & \\
\hline & Total & $55(100 \%)$ & $110(100 \%)$ & & & \\
\hline \multirow[t]{3}{*}{ Sobrepeso } & Presente & $30(54.5 \%)$ & $41(37.3 \%)$ & 0.035 & $1.04-3.89$ & 2.02 \\
\hline & Ausente & 25 (45.5\%) & $69(62.7 \%)$ & & & \\
\hline & Total & $55(100 \%)$ & $110(100 \%)$ & & & \\
\hline \multirow[t]{3}{*}{ Obesidad } & Presente & 47 (85.5\%) & $74(67.3 \%)$ & 0.013 & $1.22-6.67$ & 2.85 \\
\hline & Ausente & $8(14.55)$ & $36(32.75)$ & & & \\
\hline & Total & 55 (100\%) & 110 (100\%) & & & \\
\hline \multirow[t]{3}{*}{ Enfermedad renal crónica } & Presente & $20(36.4 \%)$ & $16(14.5 \%)$ & 0.001 & $1.56-7.20$ & 3.35 \\
\hline & Ausente & 35 (63.6\%) & 94 (85.5\%) & & & \\
\hline & Total & $55(100 \%)$ & $110(100 \%)$ & & & \\
\hline \multirow[t]{3}{*}{ Hipertensión arterial } & Presente & 37 (67.3\%) & $54(49.1 \%)$ & 0.027 & $1.08-4.19$ & 2.13 \\
\hline & Ausente & $18(32.7 \%)$ & 56 (50.9\%) & & & \\
\hline & Total & $55(100 \%)$ & $110(100 \%)$ & & & \\
\hline \multirow[t]{3}{*}{ Dislipidemia } & Presente & 27 (49.1\%) & $88(80 \%)$ & 0.000 & $0.11-0.48$ & 0.24 \\
\hline & Ausente & $28(50.9 \%)$ & $22(20 \%)$ & & & \\
\hline & Total & $55(100 \%)$ & $110(100 \%)$ & & & \\
\hline
\end{tabular}


Los factores de riesgo modificables considerados en el presente trabajo de investigación, hemoglobina glicosilada mayor de 7.5\% (mal control), onicomicosis, enfermedad vascular periférica, neuropatía periférica, antecedente de tabaquismo, obesidad, sobrepeso, enfermedad renal crónica, hipertensión arterial y dislipidemia están asociados estadísticamente a pie diabético. (Tabla3.)
Los pacientes que tienen dislipidemia tienen 0.24 veces más riesgo de desarrollar pie diabético, la asociación es estadísticamente significativa ( $p$ : 0.000) y por presenta un OR de 0.24 se comporta como un factor de protección, presenta un intervalo de confianza de $0.11-0.48$, que al no contener la unidad lo hace estadísticamente significativo.

Tabla 4. Análisis multivariado de las variables asociadas a pie diabético.

\begin{tabular}{|c|c|c|c|c|}
\hline CONDICIONES ASOCIADAS & & P VALOR & IC 95\% & OR \\
\hline \multirow{3}{*}{ Hemoglobina glicosilada } & Mal control & & & \multirow{3}{*}{4.551} \\
\hline & Buen control & 0.029 & $1.171-17.685$ & \\
\hline & Presente & 0.014 & $1.483-34.074$ & \\
\hline Onicomicosis & Ausente & & & \\
\hline \multirow[t]{3}{*}{ Enfermedad vascular periférica } & Presente & 0.229 & $0.642-6.341$ & 2.018 \\
\hline & Ausente & & & \\
\hline & Presente & 0.085 & $0.071-8.252$ & 2.681 \\
\hline Neuropatía periférica & Ausente & & & \\
\hline \multirow[t]{2}{*}{ Antecedente de tabaquismo } & Presente & 0.031 & $1.095-6.349$ & 2.666 \\
\hline & Ausente & & & \\
\hline \multirow[t]{2}{*}{ Hipertensión arterial } & Presente & 0.101 & $0.865-5.103$ & 2.101 \\
\hline & Ausente & & & \\
\hline \multirow[t]{2}{*}{ Enfermedad renal crónica } & Presente & 0.047 & $1.014-9.013$ & 3.023 \\
\hline & Ausente & & & \\
\hline \multirow[t]{2}{*}{ Tiempo de enfermedad } & $>10$ años & 0.000 & $4.121-39.608$ & 12.777 \\
\hline & $<10$ años & & & \\
\hline
\end{tabular}

En la Tabla 4, en el análisis multivariado se observa que las variables tiempo de enfermedad (OR: 12.777, IC: 4.121- 39.608, p: 0.000), hemoglobina glicosilada mal controlada (OR:4.551, IC: 1.171- 17.685, p: 0.029), onicomicosis (OR: 7.108, IC: 1.483- 34.074, p: 0.014), antecedente de tabaquismo (OR: 2.666, IC: 1.0956.349, p: 0.031 y enfermedad renal crónica como comorbilidad (OR: 3.023, IC: 1.014- 9.013, p: 0.047) están asociadas significativamente a pie diabético.

\section{DISCUSIÓN}

En el presente estudio se encontró que el sexo masculino no tiene asociación significativa para presentar pie diabético (OR: 0.86, IC: 0.45 - 1.65, p: 0.65), este resultado se contradice con el estudio de Mildred5, quién reporta que el ser hombre aumenta el riesgo de presentar pie diabético (OR: 2, IC: $1.10<$ OR < 3.72, p: 0.01528), lo mencionado se puede atribuir a que la muestra de varones fue insuficiente.
Al relacionar la edad avanzada, mayores de 60 años y el presentar pie diabético se encontró que no hay asociación significativa (OR: 0.63 IC: 0.30 - 1.33, p: 0.22), este resultado coincide con el estudio de Mildred5, quién reportó OR: 1.65 , lo que indica una asociación leve, la cual no fue respaldada por el IC: 0.84- 3.25 ni por el p que fue mayor a 0.05; se infiere que en ambos casos no se tuvo la muestra necesaria para demostrar dicha asociación.

El tiempo de evolución de la enfermedad, mayor a 10 años, presentó una asociación significativa con el desarrollo de pie diabético (OR: 2.26, IC 1.13 - 4.52, p: 0.019), comportándose como un factor de riesgo para presentar pie diabético; este resultado coincide con el estudio de Márquez-Godínez ${ }^{6}$ (OR: 5.1, IC: 95\% 2.89.4). En un estudio de Enciso ${ }^{7}$, no se corroboró una asociación significativa entre el tiempo de evolución de la enfermedad y la aparición de pie diabético ( $p$ : 0.5), del mismo modo en el estudio de Mildred ${ }^{5}$ en 
relación al tiempo de evolución de la enfermedad, no se encontró asociación entre el tiempo transcurrido desde el diagnóstico de diabetes y la presencia de pie diabético (OR: 0.96).

El porcentaje de hemoglobina glicosilada mayor a $7.5 \%$, como margen de mal control de glucemia, presentó una asociación significativa con el pie diabético (OR: 6.2, IC: 1.79 - 21.41, p: 0.001) sin embargo, en el estudio de Mildred5 no se corroboró tal asociación (OR: 1.18, IC: 0.64 < OR 2.16, p: 0.569). Márquez-Godínez6, identificó que una hemoglobina glicosilada $(\mathrm{HbA} 1 \mathrm{c}) \geq 7,0 \%$, se presenta como factor de riesgo para desarrollar pie diabético (OR: 2.8, IC: $95 \%$ 1.5-5.0).

La enfermedad vascular periférica se comportó como factor de riesgo para presentar pie diabético (OR: 2.54, IC: 1.31 - 4.94, p: 0.005). En el estudio realizado por Flores8, esta variable presentó OR: 3.75 , IC 95\% 2.73 - 5.15, p: 0.00 , comportándose como un factor de riesgo con asociación significativa. De igual modo en el estudio de Mildred5 se pudo establecer una fuerte relación con significancia estadística (OR: 13.62, IC: 6.23-30.34, $p<0.001$ )

La neuropatía periférica se comportó como factor de riesgo para el desarrollo de pie diabético (OR: 2.88, IC: 1.45 - 5.72 y p: 0.002). En el estudio de Mildred5, se demostró una asociación importante entre la neuropatía y la presencia de pie diabético (IC $<4.95<$ OR 21.02 y $\mathrm{p}<0.05)$. En el estudio de Neyra9, se encontró como principal factor de riesgo $(O R$ de $71.8, \mathrm{p}:<0.000)$ y en el estudio de Enciso7, esta variable se comportó como factor de riesgo sin embargo no fue estadísticamente significativa (OR: 2.3, IC: 95\% 0.7-7.4, p: 0.2).

Los pacientes que presentan onicomicosis tienen 4.83 veces más riesgo de desarrollar pie diabético, encontrando OR: 4.83, IC: 1.38 - 16.86 y p: 0.007. Este resultado es respaldado en el estudio de Enciso ${ }^{7}$, el cual demostró una fuerte asociación entre la presencia de onicomicosis y la posibilidad de desencadenar pie diabético (OR: 2.8, IC: 95\% 1.1- 7.2, p: 0.02).

El antecedente de tabaquismo se comportó como un factor de riesgo asociado significativamente (OR: 3.09, IC: 1.47 - 6.50, p: 0.002). En el estudio realizado por Flores8, se investigó el hábito de fumar como factor de riesgo asociado a pie diabético encontrando OR: 3.27, IC: $95 \%$ 1.27-8.39 y p: 0,01 , siendo significativamente asociado.

Con respecto a las comorbilidades como factor de riesgo asociado para pie diabético, se encontró que la hipertensión arterial y la enfermedad renal crónica, están significativamente asociadas, OR: 2.13, IC: 1.08 - 4.19, p: 0.027 y OR: 3.35 , IC: $1.56-7.20$, p: 0.0021 , respectivamente. La comorbilidad dislipidemia se comportó como un factor protector con el desarrollo de pie diabético, presentando OR: 0.24, IC: 0.11 - 0.48 y p: 0.000 . En el estudio de Chinchari10, la hipertensión arterial ( $p: 0.29$ ) y la dislipidemia ( $p$ : 0.12 ), no presentaron diferencia significativa para ser consideradas factor de riesgo.

En el estudio de Neyra9', se encontró que la hipertensión arterial se comportó como factor protector, encontrando asociación significativa (OR: $0.1, \mathrm{p}: 0,0025)$.

\section{CONCLUSIÓN}

El tiempo de enfermedad mayor a diez años es un factor de riesgo no modificable asociado a pie diabético. Además, la hemoglobina glicosilada mayor de $7.5 \%$ (mal control), la onicomicosis, el antecedente de tabaquismo y la comorbilidad enfermedad renal crónica son factores de riesgo modificables asociados a pie diabético.

La enfermedad vascular periférica, la neuropatía periférica, la hipertensión arterial no son factores de riesgo modificables asociados a pie diabético.

Contribuciones de autoría: Los autores participaron en la generación, redacción y aprobación final del artículo, además YAE en la recolección de datos.

Financiamiento: Autofinanciado.

Conflicto de interés: Los autores declaran no tener conflicto de interés en la publicación de este artículo.

Recibido: 27 de diciembre 2018

Aprobado: 15 de febrero 2019

Correspondencia: Yajaira E. Arribasplata Espinoza

Dirección: Jr. Japurá 275 dpto. 202 Breña. Lima - Perú

Teléfono: +51965462760

Correo:amyjaeli4@gmail.com 


\section{REFERENCIAS BIBLIOGRÁFICAS}

1. (1OMS). World Health Organization- Informe mundial sobre la diabetes, 2016. www.who.int/diabetes/global-report. WHO/NMH/ $\mathrm{NVI} / 16.3$.

2. (OMS). Organización Mundial de la Salud - Perfiles de los países para la diabetes,2016.http://www.who.int/diabetes/countryprofiles/ per_es.pdf?ua=1.

3. Seclén S. Diabetes Mellitus en el Perú: Hacia dónde vamos. Diabetes mellitus in Peru: Where we are going. Rev Med Hered. 2015; 26:3-4.

4. De la Cruz Vargas JA, Correa López LE, Alatrista Gutiérrez de Bambaren M del S, Sánchez Carlessi HH, Luna Muñoz C, Loo Valverde M, et al. Promoviendo la investigación en estudiantes de Medicina y elevando la producción científica en las universidades: experiencia del Curso Taller de Titulación por Tesis. Educ Médica [Internet]. 2 de agosto de 2018 [citado 16 de enero de 2019]. Disponible Sciencediret.com/science/ article/pii/S1575181318302122.

5. Mildred Rosales A, Bonilla Rojas J, Gómez Batista A, Gómez Chaparro - C, Pardo García J, Villanueva Rodríguez L. Factores asociados al pie diabético en pacientes ambulatorios. Centro de Diabetes Cardiovascular del Caribe. Barranquilla (Colombia). Salud Uninorte. Barranquilla (Col.) 2012; 28 (1): 65-74
6. Márquez-Godínez SA, Zonana-Nacach A, Anzaldo-Campos MC, Muñoz-Martínez JA. Riesgo de pie diabético en pacientes con diabetes mellitus tipo 2 en una unidad de medicina de familia. Semergen. 2014; 40(4):183-8.

7. Enciso Rojas A. Factores de riesgo asociados al pie diabético Risk factors associated with diabetic foot. Rev. virtual Soc. Parag. Med. Int. setiembre $2016 ; 3$ (2): 58-70

8. Flores R. Factores asociados al desarrollo de pie diabético en pacientes con Diabetes Mellitus tipo 2 en el servicio de Medicina Interna 03 y Pie Diabético del Hospital Nacional Guillermo Almenara Irigoyen durante el año 2012. [Tesis para optar el título profesional de Médico Cirujano]. 2013. Universidad Nacional Jorge Basadre Grohmann, Tacna.

9. Neyra-Arisméndiz L, Solís-Villanueva J, Castillo-Sayán O, GarcíaRamos F. Diabetic foot. Rev Soc Peru Med Interna 2012; vol 25 (2).

10. Chanchari-Perez L. Factores de riesgo asociados a pie diabético en pacientes atendidos en el hospital regional de Loreto. Enero 2014 diciembre 2014. Región Loreto. Iquitos - Perú. 2015.

\section{latindex}

http://www.latindex.org/latindex/ficha?folio=14280<smiles>C1=[In]CCC1</smiles>

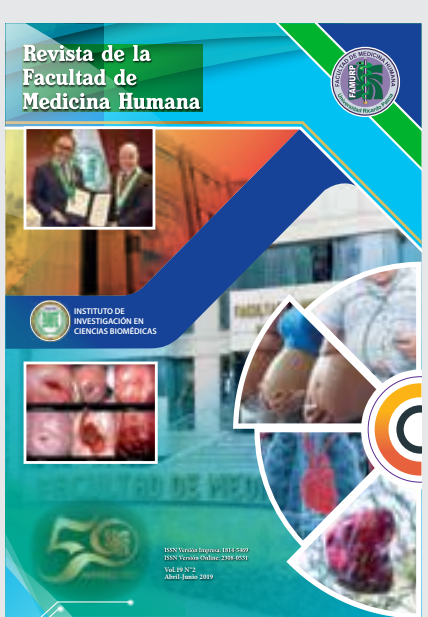

